priority. COPD palliative care provision currently focuses upon physical symptom management with less attention paid to the holistic ethos of palliation. A integrative review of existing literature across twelve databases was undertaken to explore the presence of existential suffering for those living with COPD and associated palliative care needs. 35 papers were included within the review of both qualitative and quantitative underpinnings. Thematic analysis was completed, identifying key themes explaining the impact of existential suffering:

1. 'Liminality' Patients are unsure of who they currently are and who they will become throughout disease progression.

2. 'Lamented Life' Feelings of life ceasing to exist, fuelled by grief of former life prior to illness.

3. 'Loss of Personal Liberty' - Frustration that they couldn't do activities/hobbies they once could.

4. 'Existential Isolation' Becoming isolated throughout disease progression from social and personal/family relationships. Feelings of being misunderstood had a significant impact upon low feelings of self-worth.

5. 'Life Meaning' Some patients were able to identify some life meaning and purpose, through adapting hobbies and interests to within their physical capabilities.

This integrative review has given good insight into how existential suffering manifests within this patient population. Furthermore, it has identified the need for further research to explore how life meaning and purpose is found within their daily lives, alongside the need to adapt current palliative care policy to encompass addressing existential suffering.

\section{A RENAL PALLIATIVE INTEGRATED APPROACH - IMPROVING CARE FOR HAEMODIALYSIS PATIENTS?}

Louise C Gilhooley, Jenny Bennet, Alisha Morgan. Severn Hospice, Shrewsbury and Telford NHS Trust

\subsection{6/spcare-2020-PCC.186}

Background Patients with end stage renal failure on dialysis have a high symptom burden (1) and survival on dialysis can be comparable to some cancers. (2) Increasingly it is recognised that patients with advancing age and comorbidities may benefit from a conservative approach rather than dialysis.(3) Patients with non-malignant disease often receive palliative services late or not at all.(4)

Aims Development of a monthly Renal Palliative MDT meeting alongside a Supportive Care Register (SCR) with the aim to improve renal palliative integration, education and support for patients with End Stage Renal Failure (ESRF), particularly focusing on haemodialysis (HD).

Results Data from 2018/19 collected and analysed. All deaths in patients on haemodialysis were analysed. In 2019 increasing amounts of haemodialysis patients who died had been identified and placed on SCR (48\% versus 70\%). Numbers of deaths in those not on SCR or discussed at MDT reducing. $69 \%$ of patients identified on SCR died in a community setting in 2018, this has increased in 2019 to $75 \%$ dying in a community setting.

Conclusion A SCR and monthly MDT improved access by identifying patients earlier. Numbers of deaths in those not on SCR or discussed at MDT reducing each year, suggesting we are missing less of the patients who are likely to die. Some HD deaths will be in chronic patients who we could not predict would die suddenly. The referrals have been appropriate and controlled in number. The majority of patients on SCR and discussed died out of hospital in a community setting. A greater understanding of the population we support and mutual education has resulted in closer working relationships and increased referrals. Further development of a joint Nephrology/Palliative clinic is planned. A focus group to identify patient and carer needs and experiences is planned.

\section{THE SURPRISE QUESTION IN SEVERE COPD EXACERBATIONS: CORRELATION WITH DNACPR DECISIONS AND ADVANCE CARE PLANNING}

Sarah M Gillespie, Nicholas D Lane, Katherine E Frew, Stephen C Bourke. Respiratory Research Northumbria Healthcare NHS Foundation Trust, Institute of Cellular Medicine Newcastle University, Department of Palliative Medicine Northumbria Healthcare NHS Foundation Trust, Institute of Health and Society Newcastle University

\subsection{6/spcare-2020-PCC.187}

Background Patients with COPD have a higher symptom burden than those with lung cancer. One year mortality following admission with an exacerbation of COPD (ECOPD) is 23.2\%. The 'Surprise Question' is an established screening tool designed to select patients likely to have a poor prognosis and palliative care needs. We examined whether there was a correlation between a 'No' answer to this question and evidence of prognostic or advance care planning discussions.

Methods As part of the MoSHCOPD study (clinicaltrials.gov NCT03657121) unique, consecutive patients surviving hospital admission for ECOPD were recruited. During admission, the responsible consultant was asked 'Would you be surprised if this patient died within the next year?'. On discharge, we recorded whether the patient had a Do Not Attempt Cardiopulmonary Resuscitation (DNACPR) decision in place (prognostic discussions not resulting in a DNACPR decision were not captured) and whether there was evidence of further advance care planning or specialist palliative care (SPC) input.

Results Of 314 patients recruited to date, a 'No' answer to the Surprise Question was given in 151 (48.9\%). Of these, 87 (57.6\%) had a DNACPR decision recorded on discharge and 15 (9.9\%) had evidence of either advance care planning or SPC involvement. In patients with both a 'No' answer and an active DNACPR, 15 (17.2\%) had evidence of either advance care planning or SPC involvement.

Conclusion Despite clinicians having a pessimistic expectation of one year survival in this patient group, rates of advance care planning and SPC involvement are disappointingly low, in keeping with previous research in this area. Clinicians' lack of confidence in estimating prognosis following ECOPD is likely to contribute to these findings and needs to be urgently addressed. The MoSHCOPD study aims to improve prognostication following ECOPD, thus supporting clinicians to engage in advance care planning in this underserved patient group. 